PROCEEDINGS OF THE

AMERICAN MATHEMATICAL SOCIETY

Volume 124, Number 11, November 1996

\title{
THE STANDARD CANTOR FUNCTION IS SUBADDITIVE
}

\author{
JOZEF DOBOŠ
}

(Communicated by Andreas R. Blass)

\begin{abstract}
In this paper the subadditivity of the Cantor function $\phi:[0,1] \rightarrow$
\end{abstract} $[0,1]$ is proved.

Let us begin by recalling that a function $f: \mathbb{R} \longrightarrow \mathbb{R}$ is said to be subadditive, if it satisfies the inequality $f(x+y) \leqq f(x)+f(y)$ whenever $x, y \in \mathbb{R}$. (See [2] and [4].)

The usual definition of the standard Cantor function involves the classic middlethirds description of the standard Cantor set. (See [1] and [3].) We offer an alternate definition of this function.

Define a sequence of functions $\phi_{n}: \mathbb{R} \longrightarrow[0,1]$ by

$$
\phi_{0}(x)=\left\{\begin{array}{ll}
0 & \text { if } x \leq 0, \\
x & \text { if } 0 \leq x \leq 1, \\
1 & \text { if } x \geq 1,
\end{array} \quad \phi_{n+1}(x)= \begin{cases}\frac{1}{2} \cdot \phi_{n}(3 x) & \text { if } x \leq \frac{2}{3}, \\
\frac{1}{2}+\frac{1}{2} \cdot \phi_{n}(3 x-2) & \text { if } x \geq \frac{1}{3} .\end{cases}\right.
$$

It is easy to check that each $\phi_{n}$ is non-decreasing, that $\phi_{n}(x)=0$ for all $x \leq 0$, that $\phi_{n}(x)=1$ for all $x \geq 1$, and that the two lines in the definition of $\phi_{n+1}$ agree in the overlap of their domains, both giving $\phi_{n+1}(x)=\frac{1}{2}$ when $\frac{1}{3} \leq x \leq \frac{2}{3}$.

Put $\phi=\lim _{n \rightarrow+\infty} \phi_{n}$. It is not difficult to verify that the restriction of $\phi$ to $[0,1]$ is the standard Cantor function. The functions $\phi_{n}$ are polygonal approximations of $\phi$.

Theorem. The standard Cantor function is subadditive.

Proof. The function $\phi$ is the pointwise limit of the functions $\phi_{n}$ as $n \rightarrow+\infty$. So to prove the subadditivity of $\phi$, it suffices to prove the subadditivity of all $\phi_{n}$, which we do by induction on $n$. The case $n=0$ is trivial, so we proceed to the induction step from $n$ to $n+1$. Let $x, y \in \mathbb{R}, x \geq y$. Here we consider several cases.

Case 1: $y \leq 0$. This case is trivial as $f_{n+1}$ is monotone.

Case 2: $y \geq \frac{1}{3}$. In this case,

$$
\phi_{n+1}(x+y) \leq 1=\frac{1}{2}+\frac{1}{2} \leq \phi_{n+1}(x)+\phi_{n+1}(y) .
$$

Case 3: $x \leq \frac{1}{3}$. As $x, y$ and $x+y$ are all $\leq \frac{2}{3}$, we have

$$
\begin{aligned}
\phi_{n+1}(x+y) & =\frac{1}{2} \cdot \phi_{n}(3 x+3 y) \\
& \leq \frac{1}{2} \cdot \phi_{n}(3 x)+\frac{1}{2} \cdot \phi_{n}(3 y)=\phi_{n+1}(x)+\phi_{n+1}(y) .
\end{aligned}
$$

Received by the editors September 2, 1994 and, in revised form, May 11, 1995.

1991 Mathematics Subject Classification. Primary 26D15.

Key words and phrases. Subadditive functions. 
Case 4: $0 \leq y \leq \frac{1}{3} \leq x$. As $x+y \geq \frac{1}{3}$, we have

$$
\begin{aligned}
\phi_{n+1}(x+y) & =\frac{1}{2}+\frac{1}{2} \cdot \phi_{n}(3 x+3 y-2) \\
& \leq \frac{1}{2}+\frac{1}{2} \cdot \phi_{n}(3 x-2)+\frac{1}{2} \cdot \phi_{n}(3 y)=\phi_{n+1}(x)+\phi_{n+1}(y) .
\end{aligned}
$$

These four cases exhaust all the possibilities, so the proof is complete.

Let us recall that a modulus of continuity is a function $f$ defined, continuous, nondecreasing and subadditive on $[0,1]$ with $f(0)=0$.

Corollary. The standard Cantor function is a modulus of continuity.

The author wishes to thank the referee for his useful suggestions.

\section{REFERENCES}

1. Darst, R.B., Some Cantor sets and Cantor functions, Math. Mag. 45 (1972), 2-7. MR 46:311

2. Matkowski, J. and Świątkowski, T., On subadditive functions, Proc. Amer. Math. Soc. 119 (1993), 187-197. MR 93k:26002

3. Richey, M., Mapping the Cantor set onto $[0,1]$ : a new construction, preprint.

4. Rosenbaum, R.A., Subadditive functions, Duke Math. J. 17 (1950), 227-247. MR 12:164a

Department of Mathematics, Technical University, 04200 Košice, Slovakia

E-mail address: dobos@ccsun.tuke.sk 